Original Research

\title{
Diagnostic performance of arterial spin-labeled perfusion imaging and diffusion-weighted imaging in full-term neonatal hypoxic-ischemic encephalopathy
}

\author{
Linlin Meng ${ }^{1}$, Qian Wang ${ }^{2}$, Yifang $\mathrm{Li}^{3}$, Xiangxing $\mathrm{Ma}^{2}$, Wen Liं ${ }^{4}$, Qing Wang ${ }^{2, *}$ \\ ${ }^{1}$ Department of Radiology, The Second Hospital of Shandong University, 250033 Jinan, Shandong, China \\ ${ }^{2}$ Department of Radiology, Qilu Hospital of Shandong University, 250012 Jinan, Shandong, China \\ ${ }^{3}$ Department of Radiology, Rizhao People's Hospital, 276800 Rizhao, Shandong, China \\ ${ }^{4}$ Department of Pediatrics, Qilu Hospital of Shandong University, 250012 Jinan, Shandong, China \\ *Correspondence: 2019120135@mail.sdu.edu.cn; qlradiology@163.com (Qing Wang)
}

DOI:10.31083/j.jin2004099

This is an open access article under the CC BY 4.0 license (https://creativecommons.org/licenses/by/4.0/).

Submitted: 6 September 2021 Revised: 29 November 2021 Accepted: 6 December 2021 Published: 30 December 2021

We evaluated the performance of arterial spin-labeled perfusion imaging and diffusion-weighted imaging in diagnosing full-term neonatal hypoxic-ischemic encephalopathy. Arterial spin-labeled, diffusion-weighted imaging and conventional magnetic resonance imaging (T1-weighted imaging, T2-weighted imaging and $T_{2}$ fluidattenuated inversion recovery) were performed in 23 full-term neonates with hypoxic-ischemic encephalopathy group 10 normal neonates (Control group). The cerebral blood flow and the apparent diffusion coefficient were measured in the bilateral basal ganglia, thalamus and frontal white matter. The effect of neonatal age on the CBF and apparent diffusion coefficient values were further investigated after dividing the 23 ischemic encephalopathy cases into three subgroups ( $1-3$ days, 4-7 days, and 8-15 days). The cerebral blood flow values in the thalamus and lenticular nucleus were significantly higher. The apparent diffusion coefficient values in the thalamus, frontal white matter and lenticular nucleus head were significantly lower in the hypoxic-ischemic encephalopathy group than those in the Control group $(p<0.05)$. There were no significant differences between the ischemic encephalopathy and Control groups in the cerebral blood flow values in the caudate nucleus head and frontal lobe white matter $(p>0.05)$. The cerebral blood flow and apparent diffusion coefficient values in the thalamus and lenticular nucleus were negatively correlated. Comparison among different age subgroups of hypoxic-ischemic encephalopathyneonates showed that the cerebral blood flow value was higher. In comparison, the apparent diffusion coefficient value was lower in the 1-3 days old neonates than those in the older neonates $(p<0.05)$. Arterial spin-labeled and diffusion-weighted imaging could reflect the ischemic encephalopathy pathological processes more comprehensively. The cerebral blood flow measurement and apparent diffusion coefficient values in the thalamus and the lenticular nucleus may represent a novel way to diagnose ischemic encephalopathy early.

\section{Keywords}

Hypoxic-ischemic encephalopathy; Neonate; Magnetic resonance imaging; Arterial spin labeling; Diffusion-weighted imaging

\section{Introduction}

Hypoxic-ischemic encephalopathy (HIE) is a common reason for the early death of neonates. HIE occurs in 1 to 8 per 1000 live births in developed countries [1]. Some asphyxiated newborns have decreased cerebral blood flow (CBF) on the first day of life, which may be related to the development of their brains and hypoxic-ischemic injury, and hyperperfusion usually reaches the peak on days $2-3$ of their lives [2, 3].

Magnetic resonance imaging (MRI) is the preferred method for HIE diagnosis. The water content in brain tissue increases after hypoxia, leading to prolonging $\mathrm{T} 1$ and T2 values on the MR image [4]. Studies have shown that T1-weighted (T1WI) and T2-weighted (T2WI) imaging is sensitive to the pathological processes of neuronal necrosis and water changes in neonates of 3-7 days and 6-10 days, respectively $[4,5]$. In clinical practice, dynamic susceptibility contrast-enhanced perfusion MRI is commonly used to evaluate the microcirculation of brain tissue. With this technique, the hemodynamics, such as $\mathrm{CBF}$, mean transit time, cerebral blood volume, and time to peak, can be evaluated through the time-signal intensity curve following the administration of contrast agents. However, its application in pediatrics is limited because of the administration of contrast agents, and few studies are using perfusion-weighted imaging in the very immature brain $[6,7]$.

Arterial spin labeling (ASL) is a new MR technique by which the regional CBF can be quantified using CBF images. It has been reported that ASL can evaluate abnormal brain perfusion in neonatal brain injury [8, 9]. Diffusion-weighted imaging (DWI) is to date the only non-invasive method to detect water molecule diffusion in vivo. Compared with the conventional MRI, DWI can detect the pathological process of early hypoxic-ischemic lesions and quantitatively measure the apparent diffusion coefficient (ADC) [10-13], which is positively related to the diffusion rates of water molecules. In 
the case of restricted diffusion, the signal intensity exhibits an increase on the DWI image and a decrease on the ADC map.

We measured the $\mathrm{CBF}$ and ADC values of the full-term HIE neonates to determine the diagnostic performance of ASL and DWI for HIE.

\section{Materials and methods \\ 2.1 Patients}

Neonates who were clinically diagnosed with HIE and underwent MRI examination from October 2018 to August 2020 were enrolled. They were diagnosed with HIE according to the HIE diagnostic criteria developed by the American Society of Obstetrics and Gynecology (ACOG) in 2014 [14]. The main causes for the HIE included birth asphyxia, cord entanglement, aspiration pneumonia, and the application of obstetric forceps or ventouse extraction for birth. Inclusion criteria: (a) gestational age $>38$ weeks; (b) age was within 15 days; (c) availability of MR DWI and ASL images. Exclusion criteria: (a) acute encephalopathy resulting from causes other than hypoxic-ischemic events, i.e., chromosomal abnormality, electrolyte abnormality, hypoglycemia, metabolic disease, neurocutaneous syndrome, neuromuscular disease, idiopathic stroke, intracranial hemorrhage, and central nervous system infection; (b) suffering from congenital brain malformation, bleeding diathesis, prolonged neonatal pulmonary hypertension, and imminent death; (c) the image quality was unsatisfying with susceptibility or motion artifacts. The HIE cases were further divided into three subgroups based on their ages (i.e., 1-3 days, 4-7 days, and 815 days) to determine the influence of age on the CBF and ADC values. During the same period, full-term newborn infants who underwent MRI for non-neurologic conditions (including icterus, esophageal atresia or facial vascular malformations without intracranial involvement) were included in the Control group. They were without any signs of encephalopathy and were with normal brain MRI images. The research was approved by the Ethics Committee (approval no. 12184). Informed consent was obtained from the parents of all neonates.

\subsection{MRI sequences and imaging parameters}

Oral chloral hydrate $(5 \%, 50 \mathrm{mg} / \mathrm{kg})$ was used for sedation 15-20 minutes ( $\mathrm{min}$ ) before the MRI examination. The cotton ball was plugged into neonates' ears for hearing protection. Heart rate and oxygen saturation were monitored during MR imaging by an intensive care neonatologist. The neonates were kept warm during the MRI examination. Magnetic field intensity was 3.0-T, and the scanner was equipped with an 8-channel head coil (Verio, postprocessing workstation, syngo MMWP VE36A, Siemens, Germany). The brain MRI scans included the axial T1WI, T2WI, T2FLAIR, sagittal T2WI, ASL, and DWI. Q2TIPS (QUIPSS II with thin-slice TI1 periodic saturation) perfusion pattern based on PICORE (proximal inversion with control for off-resonance effects) labeled scheme was adopted for the ASL. The imaging parameters of ASL were TR $=4000 \mathrm{~ms}$,
$\mathrm{TE}=17 \mathrm{~ms}$, slice thickness $=6 \mathrm{~mm}$, interslice gap $=0.6 \mathrm{~mm}$, slice number $=4$, labeling pulse flip angle $=90^{\circ}$, bandwidth $=1002 \mathrm{~Hz}$, excitation $=1$, TI $1=700 \mathrm{~ms}$, and TI $=1400 \mathrm{~ms}$. Scanning ranged from the thalamus to the level of the superior edge of the lateral ventricle with an acquisition number of 51, and the scan duration was $3 \mathrm{~min}$ and $48 \mathrm{sec}$. Echo planar imaging sequence was applied for DWI with TR $=6600$ $\mathrm{ms}, \mathrm{TE}=100 \mathrm{~ms}$, gradient magnetic field from the three directions of $X, Y$, and $Z$-axis, b value $=0,1000 \mathrm{~s} / \mathrm{mm}^{2}$, slice thickness $=6 \mathrm{~mm}$, number of excitations $=3$, and scan duration of $1 \mathrm{~min}$ and $20 \mathrm{sec}$.

\subsection{Image analysis and data process}

Two pediatric neuroradiologists (with 12 years and 16 years of experience, respectively) blinded to the grouping information reviewed the MRI data. Disagreement was resolved through discussion. ADC and CBF quantitative maps were generated online via vendor-sourced software and displayed as DICOMS in PACS. The ADC maps in the same geometry as the ASL maps were acquired. The colors of the CBF map were regulated manually in a post-processing workstation (Syngo MMWP VE36A). On the ADC maps, the bilateral caudate nucleus head, lentiform nucleus, thalamus, frontal and parietal white matter (at the level of the superior edge of the lateral ventricle) were selected as the region of interest (ROI), and the ADC was measured. The cerebrospinal fluid area was avoided from the edge of ROI, and the size of ROI was set depending on the location of the ROIs [3]. The ROI on the CBF map was determined by drawing on the original ASL images (Fig. 1). The ROIs on the CBF maps were consistent with those on the ADC maps. The CBF and ADC values between the HIE and Control groups and the relationship between the CBF and ADC were analyzed.

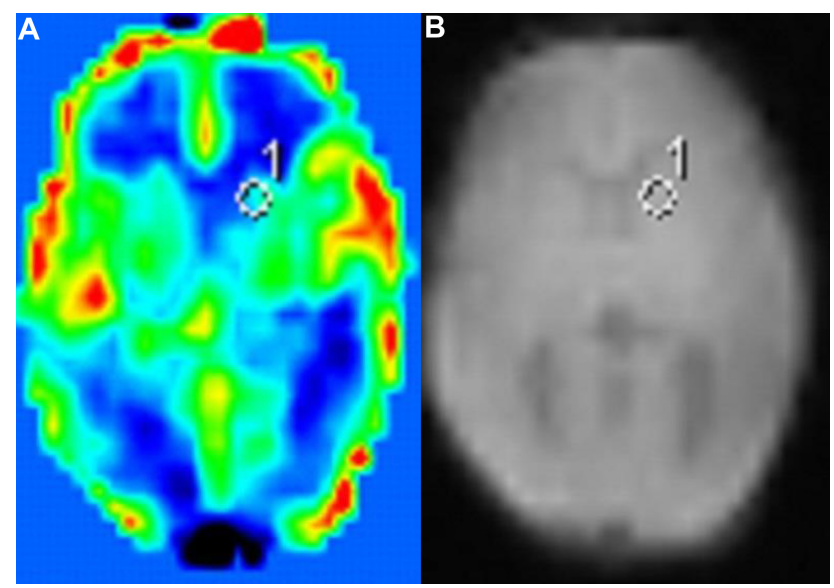

Fig. 1. ASL perfusion-weighted imaging of a control neonate. (A) CBF image. (B) The label source ASL image. The circled area indicates the region of interest (ROI) at the left caudate nucleus head level. 
Table 1. Normal and abnormal performance in conventional MRI and DWI of neonates in the HIE group.

\begin{tabular}{lcccc}
\hline Location & $\begin{array}{c}\text { Abnormal signal on } \\
\text { conventional MRI and } \\
\text { abnormal signal on DWI image }\end{array}$ & $\begin{array}{c}\text { Abnormal signal on } \\
\text { conventional MRI and normal } \\
\text { signal on DWI image }\end{array}$ & $\begin{array}{c}\text { Normal signal on conventional } \\
\text { MRI and abnormal signal on } \\
\text { DWI image }\end{array}$ & $\begin{array}{c}\text { Normal signal on conventional } \\
\text { MRI and normal signal on } \\
\text { DWI image }\end{array}$ \\
\hline Lentiform nucleus & 2 & 1 & 1 & 0 \\
Thalamus & 1 & 0 & 3 & 3 \\
Frontal white matter & 2 & 0 & 7 & 3 \\
Parietal white matter & 0 & 5 & 6 \\
Total & 5 & 4 & 3 \\
\hline
\end{tabular}

\subsection{Statistical analysis}

Data were analyzed with the SPSS software (version 19.0, SPSS Inc., Chicago, IL, USA). Data are expressed as mean \pm standard deviation (SD). Two independent samples $t$-test was used to compare the CBF and ADC values between the HIE and Control groups. Pearson correlation was used to assess the relationship between $\mathrm{CBF}$ and ADC. One-way analysis of variance (ANOVA) and SNK-q test were used to analyze the influence of age on CBF and ADC. ROC curves calculated sensitivity and specificity. $p<0.05$ was considered to be statistically significant.

\section{Results \\ 3.1 Clinical characteristics}

There were 23 full-term neonates $(10$ males and 13 females) in the HIE group and 10 full-term neonates ( 6 males and 4 females) in the Control group. Their age was from 1 to 15 days. The median age was 9.1 days in the HIE group and 10.3 days in the Control group. The gestational age range in the HIE group was 37-41 weeks, with an average of 40.2 weeks, and in the Control group was 37-42 weeks, with an average of 39.7 weeks. The average weight was $2.8 \mathrm{~kg}$ in the HIE group and $3.1 \mathrm{~kg}$ in the Control group. For the HIE cases, 9 patients suffered from acute asphyxia and the other $14 \mathrm{pa}-$ tients suffered from prolonged asphyxia. Intracranial bleeding occurred in 5 cases. There were no significant differences between the HIE and Control groups regarding sex, age, gestational age, and weight.

\subsection{Sensitivity and specificity of conventional MRI, ASL, and} $D W I$ in the diagnosis of HIE

The sensitivity and specificity were measured to determine the different diagnostic values of conventional MRI, ASL and DWI in HIE. There was a consensus read between two neuroradiologists. Of the 23 cases with HIE, 10 were positive on conventional T1WI, T2WI, and T2FLAIR (sensitivity: $41.6 \%$; specificity: $54.5 \%$ ). The lesions detected with conventional MRI were located in the lentiform nucleus in 3 cases, the frontal white matter in 6 cases, and the thalamus in 1 case, exhibiting hyperintensity on T1WI and T2FLAIR hypointensity on T2WI. On the DWI and ADC maps, 12 cases showed abnormal signal intensity in the frontal and parietal white matter, thalamus, or lentiform nucleus. The distribution of the cases is shown in Table 1. The sensitivity and specificity of DWI were $50.0 \%$ and $45.4 \%$, respectively.
The CBF maps displayed CBF in the gray matter, white matter, and deep nuclei in the Control group. The CBF in the gray matter was significantly higher than that in the white matter. The blood flow in the basal ganglia was between the levels in the gray and white matter (Fig. 2). In the HIE group, hypoperfusion areas in the CBF maps were identified in the frontal lobe, parietal lobe, or basal ganglia in 5 cases, and no significant change was observed in the other 18 cases (Figs. 3,4). The sensitivity and specificity of ASL were 33.3\% and $90.9 \%$, respectively. These results indicate that ASL has a high specificity and a low sensitivity.

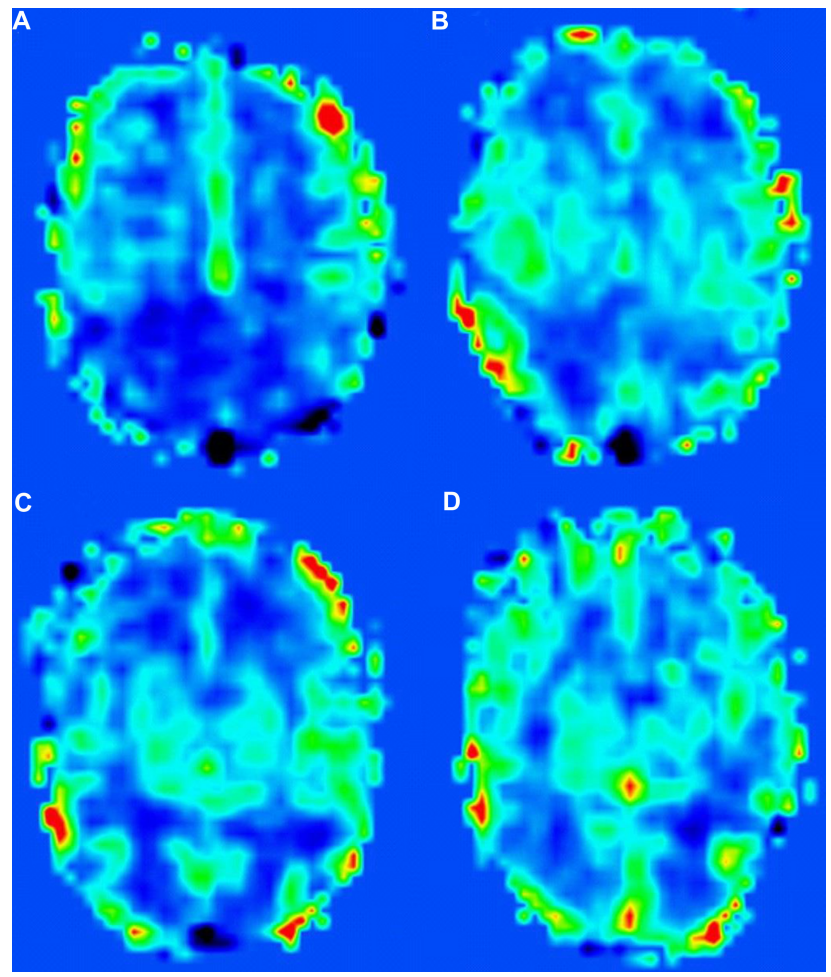

Fig. 2. The CBF images in different slices of a control neonate (7 days old, gestational age of 38 weeks and 4 days, and weight of $4.3 \mathrm{~kg}$ ). (AD) CBF images showed different blood perfusion in different areas. The red areas in the cerebral cortex indicate high perfusion. The blue areas in the white matter illustrate low perfusion. The green areas in the basal ganglia region show the perfusion between red and blue. 


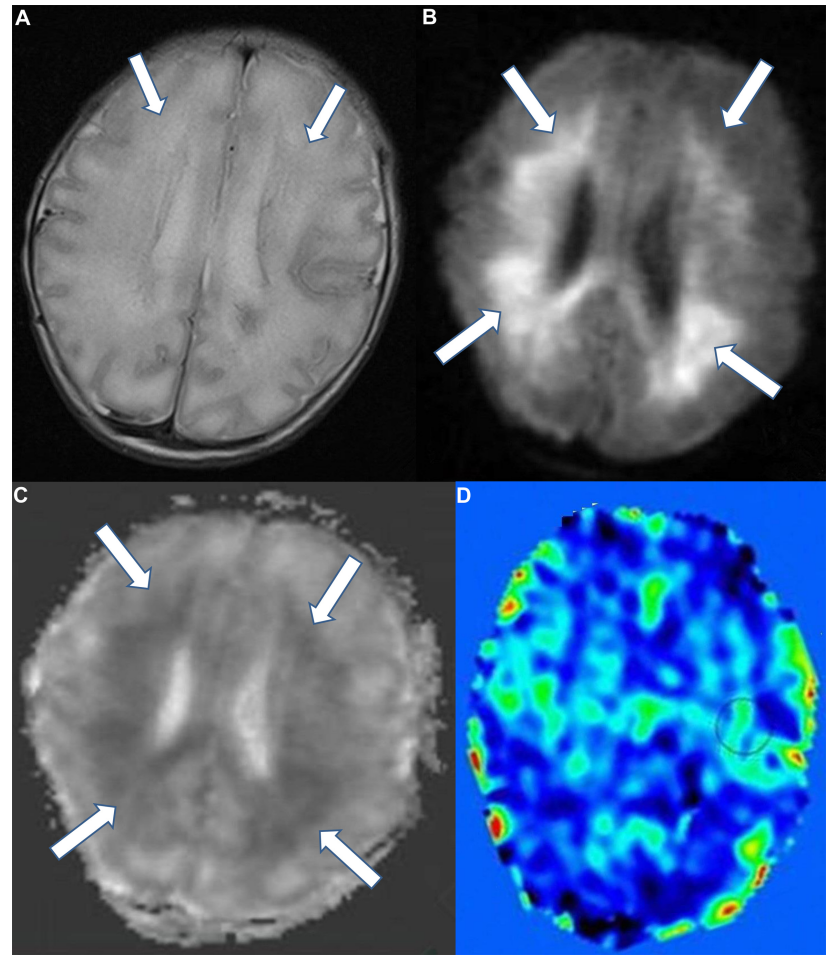

Fig. 3. Images of a male HIE neonate (3 days old, gestational age of 37 weeks and 6 days, and weight of $2.45 \mathrm{~kg}$ ) with a history of perinatal asphyxia due to an intertwined umbilical cord. (A) T2WI showed hyperintensity at the bilateral corona radiata area (arrows). (B) DWI showed hyperintensity in the same regions (arrows). (C) ADC map showed hypointensity in the same regions (arrows). (D) No abnormal perfusion was observed on the CBF images, but the CBF value in the circled area was higher than that of the control group.

\subsection{Significance of $C B F$ and $A D C$ values}

To determine the diagnostic value of ASL and DWI in $\mathrm{HIE}$, the quantitative values of $\mathrm{CBF}$ and ADC were measured. Tables 2,3 showed the CBF and ADC values of the bilateral caudate nucleus head, lentiform nucleus, thalamus, and frontal white matter. The CBF values in the thalamus and lentiform nucleus in the HIE group were significantly higher than those in the Control group $(p<0.05)$. There were no statistical differences between the two groups' CBF values in the caudate nucleus head and frontal white matter $(p>0.05)$. In contrast to $\mathrm{CBF}$ values, the ADC values in the thalamus, frontal white matter and lentiform nucleus in the HIE group were significantly lower than those in the Control group ( $p$ $<0.05$ ). Similar to CBF values, there was no significant difference for the ADC value in the caudate nucleus head between the two groups $(p>0.05)$. Pearson correlation analysis showed that the CBF and ADC values were negatively correlated in both thalamus and lentiform nucleus $(R=-0.355, p$ $<0.01$; and $R=-0.471, p=0.02$; respectively). There was no significant correlation between the CBF and ADC values in the frontal white matter and caudate nucleus head $(R=0.028$, $p=0.91 ;$ and $R=0.065, p=0.34$, respectively).

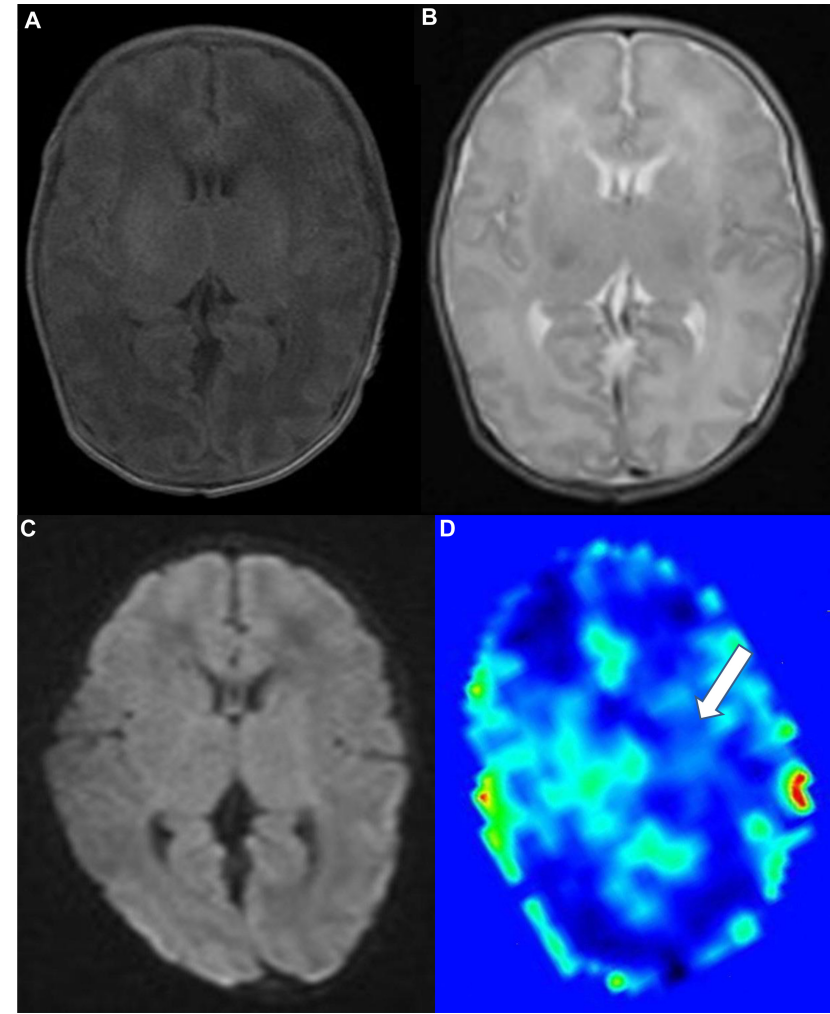

Fig. 4. Images of a 10-day old female HIE infant (with a history of intrauterine infection and neonatal asphyxia). There was no signal abnormality on T1WI (A), T2WI (B) and DWI images (C). (D) The perfusion was reduced at the left basal ganglia region on CBF maps (arrow).

\subsection{The effect of age on the CBF and $A D C$ values in the HIE neonates}

Based on their age, the 23 HIE cases were divided into three subgroups of 1-3 days, 4-7 days, and 8-15 days, based on their age to determine the effect of age on CBF and ADC. The CBF values in all analyzed areas were significantly higher. In comparison, the ADC values were significantly lower in the group of 1-3 days than those in the other two groups ( $p<0.05$, Tables 4,5$)$. There were no significant differences for both CBF and ADC values in the analyzed areas between the groups of 4-7 days and 8-15 days ( $p>0.05)$.

\section{Discussion}

ASL is an MR perfusion imaging method, first proposed and applied in animal experiments by Detre et al. in 1992 [15]. It was used in evaluating human brain perfusion for the first time in 1994 by Roberts et al. [16]. ASL employs magnetic marked arterial blood as an endogenous contrast medium and involves pulsed arterial spin labeling (PASL) or continuous arterial spin labeling (CASL) [17]. We adopted PASL technology, safe, non-invasive, convenient, no need for exogenous contrast agent, allowing repeated measurements, and less post-processing. However, PASL is relatively limited by the low signal-to-noise ratio (SNR) and poor spatial resolution. The CBF is lower, and the blood water content is higher in neonates than in adults, resulting in a higher 
Table 2. The average CBF values in different brain areas of neonates (mean $\pm \mathrm{SD}$ ).

\begin{tabular}{lcccc}
\hline \multirow{2}{*}{ ROI } & \multicolumn{4}{c}{ Average CBF value $(\mathrm{mL} / 100 \mathrm{~g} / \mathrm{min})$} \\
\cline { 2 - 5 } & HIE group $(\mathrm{n}=23)$ & Control group $(\mathrm{n}=10)$ & $t$ & $p$ value \\
\hline Caudate nucleus head & $28.33 \pm 13.42$ & $30.73 \pm 22.20$ & -0.66 & 0.522 \\
Lentiform nucleus & $55.34 \pm 24.16$ & $47.47 \pm 23.93$ & 2.74 & 0.010 \\
Thalamus & $51.96 \pm 42.24$ & $42.04 \pm 26.74$ & 2.93 & 0.004 \\
Frontal white matter & $20.51 \pm 11.29$ & $20.52 \pm 10.17$ & -0.14 & 0.198 \\
\hline
\end{tabular}

Table 3. The average ADC values in different brain areas of neonates (mean $\pm \mathrm{SD}$ ).

\begin{tabular}{lcccc}
\hline \multirow{2}{*}{ ROI } & \multicolumn{4}{c}{ Average ADC value $\left(\times 10^{-3} \mathrm{~mm}^{2} / \mathrm{s}\right)$} \\
\cline { 2 - 5 } & HIE group $(\mathrm{n}=23)$ & Control group $(\mathrm{n}=10)$ & $t$ & $p$ value \\
\hline Caudate nucleus head & $1.27 \pm 0.05$ & $1.23 \pm 0.11$ & 0.16 & 0.87 \\
Lentiform nucleus & $1.21 \pm 0.05$ & $1.25 \pm 0.07$ & 2.25 & 0.04 \\
Thalamus & $1.12 \pm 0.07$ & $1.16 \pm 0.12$ & 2.44 & 0.03 \\
Frontal white matter & $1.43 \pm 0.11$ & $1.50 \pm 0.16$ & 2.39 & 0.03 \\
\hline
\end{tabular}

Table 4. The CBF values in different age groups of HIE neonates.

\begin{tabular}{lcccc}
\hline \multirow{2}{*}{ Age (days) } & \multicolumn{3}{c}{ The average CBF value $(\mathrm{mL} / 100 \mathrm{~g} / \mathrm{min})$} \\
\cline { 2 - 5 } & Caudate nucleus head & Lentiform nucleus & Thalamus & Frontal white matter \\
\hline 1 to $3(\mathrm{n}=9)$ & $30.19 \pm 12.74$ & $57.49 \pm 23.65$ & $53.28 \pm 24.39$ & $26.54 \pm 14.76$ \\
4 to $7(\mathrm{n}=12)$ & $21.05 \pm 11.26$ & $49.36 \pm 21.47$ & $47.39 \pm 20.74$ & $21.87 \pm 10.43$ \\
8 to $15(\mathrm{n}=2)$ & $27.54 \pm 16.83$ & $52.13 \pm 27.32$ & $44.93 \pm 25.08$ & $22.75 \pm 12.66$ \\
$F$ value & 3.799 & 6.915 & 4.763 & 5.625 \\
$p$ value & 0.041 & 0.006 & 0.021 & 0.013 \\
\hline
\end{tabular}

Table 5. The ADC values in different age groups of HIE neonates.

\begin{tabular}{lcccc}
\hline \multirow{2}{*}{ Age (days) } & \multicolumn{4}{c}{ The average ADC values $\left(\times 10^{-3} \mathrm{~mm}^{2} / \mathrm{s}\right)$} \\
\cline { 2 - 5 } & Caudate nucleus head & Lentiform nucleus & Thalamus & Frontal white matter \\
\hline 1 to $3(\mathrm{n}=9)$ & $0.987 \pm 0.086$ & $1.169 \pm 0.124$ & $1.104 \pm 0.055$ & $1.403 \pm 0.261$ \\
4 to $7(\mathrm{n}=12)$ & $1.208 \pm 0.103$ & $1.486 \pm 0.073$ & $1.625 \pm 0.232$ & $1.874 \pm 0.433$ \\
8 to $15(\mathrm{n}=2)$ & $1.315 \pm 0.139$ & $1.465 \pm 0.071$ & $1.817 \pm 0.142$ & $1.727 \pm 0.079$ \\
$F$ value & 4.145 & 4.970 & 4.383 & 5.168 \\
$p$ value & 0.031 & 0.018 & 0.026 & 0.016 \\
\hline
\end{tabular}

T1 value and lower SNR [18-20]. Although CASL has been shown to generate a higher SNR and provide a better perfusion contrast than PASL, it may lead to neonatal radiofrequency burn because of the high specific absorption rate [21]. In addition, CASL may overestimate the perfusion because its longer labeled pulse could produce a magnetization transfer effect. Little is known for recently developed ASL methods such as pseudo-continuous arterial spin labeling for their application in pediatrics due to their complexity [22]. The PASL was used to measure the $\mathrm{CBF}$, showing a sensitivity of $33.3 \%$ and a specificity of $90.9 \%$ in the diagnosis of HIE. The sensitivity of CBF was lower than that of conventional MRI and DWI (41.6\% and 50\%, respectively), which may be due to the poor resolution of PASL. The low sensitivity and specificity for DWI are likely due to the wide time range of the MRI.

Additionally, the sensitivity and specificity of ASL were different in our work, which was carried out by de Vis et al. [9]. The sensitivity was $85.7 \%$, and the specificity was $100 \%$. This may be due to the performance time of MRI. The MRI was performed within 15 days after birth, while, in de Vis et al. [9] results, the MRI was performed 2-7 days after birth.

The HIE lesions in 7 cases showed high signal intensity on DWI images and low signal intensity on ADC maps. In contrast, their signal intensity was normal in the conventional T1WI, T2WI and T2FLAIR sequences. These results indicated that DWI was more sensitive to the pathological changes in HIE than conventional MRI sequences, which is consistent with previous studies [12, 23]. Previous studies have reported that brain perfusion may be affected by developmental and postnatal age [16-18]. Reduced CBF and oxygen supply may result in a series of biochemical reactions and ultimately lead to cell damage in HIE [24]. For acute cell injury, the diffusion rate of water molecules in cells may reduce due to cytotoxic edema, thus showing decreased ADC values and high DWI signal intensity [25]. At the early stage of HIE, conventional MRI sequences may not detect the lesions because there is neither a significant change of the water molecule content nor necrosis in the damaged brain tissue. 
However, 5 HIE cases showed no abnormalities on DWI images and ADC maps but the abnormal signal intensity in conventional MRI sequences. This result may be explained that the hypoxic-ischemic lesion may be in the subacute or early recovery stage. In this stage, selective necrosis of the cortex and proliferation of glial cells can cause the increase of water molecule diffusion and ADC values. Thus the high signal intensity in the infarction area may gradually develop into an isointensity signal on DWI images $[12,26]$. Another possible explanation might be that the lesions may be in the period of ADC pseudonormalization. The cerebral vessels could automatically adjust the blood flow in 5 to 10 days, which may cause reperfusion injury and blood-brain barrier damage [27]. Therefore, water molecular diffusion increases and the ADC values return to normal [28]. Winter et al. [29] have demonstrated that ADC may show the "pseudonormalization" phenomenon in the HIE neonates 10 days from birth in the areas of frontal and occipital white matter, thalamus, lentiform nucleus, and posterior limb of the internal capsule. During this period, DWI may not exhibit abnormal signal intensity.

Comparative analysis showed that the CBF values in the thalamus and lentiform nucleus were higher. The ADC values in the thalamus, frontal white matter, and lentiform nucleus were lower in the HIE cases than those in the Controls. The CBF and ADC values changes in the thalamus and lentiform nucleus were more significant than in the other regions. There was a negative correlation between the $\mathrm{CBF}$ and $\mathrm{ADC}$ values. These results indicate that the $\mathrm{CBF}$ and $\mathrm{ADC}$ in the hypothalamus and lentiform nucleus are more sensitive to the pathological processes of ischemia and hypoxia. The hypothalamus and lentiform nucleus vessels may be dilated, accompanied by short-term high perfusion and restricted diffusion of water molecules [30]. Pienaar et al. [31] have also shown that the decreased ADC value was correlated with increased $\mathrm{CBF}$ value in the hypoxic-ischemic region, consistent with our results. These indicate that ASL and DWI can help early diagnosis of HIE.

In addition, the CBF value in HIE neonates aged 1-3 days was higher than those in elder HIE neonates, but there was no significant difference among neonates aged 4 days and older. The hypoxic-ischemic cerebral injury is acute in the HIE neonates aged 1-3 days, and the blood flow compensation increases [3]. However, three days later, the brain tissue is already at the stage of ischemia-reperfusion, and autoregulation of cerebral blood vessels is decompensated, leading to reduced cerebral blood flow and CBF values [3]. Therefore, CBF might reflect the pathological process of brain injury in HIE, which is consistent with the previous report [31]. Aslan et al. [32] have demonstrated that regional CBF was more sensitive in detecting small CBF changes than absolute CBF. In addition, the ADC values in the HIE neonates aged 1-3 days were also lower than those in the other age groups. Barkovich et al. [11] have suggested that the DWI sequence was sensitive to cytotoxic edema induced by early cerebral is- chemia and could reflect the severity and range of brain injury earlier than conventional MRI sequences. They also observed that some HIE cases showed decreased ADC values but no abnormal findings on the DWI images, indicating that the $\mathrm{ADC}$ value measurement is more accurate and sensitive than the DWI image. These indicate that $\mathrm{CBF}$ and $\mathrm{ADC}$ values are important in the 1st-3rd days of life. Liauw et al. [33] and Rutherford et al. [34] have obtained similar results, showing reduced ADC values but no abnormal signal intensity on DWI images in some hypoxic-ischemic lesions. Nevertheless, it was noticed that the scan range of ASL was small and the scan time was relatively long, so that the temporal and spatial resolution of pulse ASL is relatively low.

We encountered some limitations apart from the small sample size, and there was a low sensitivity and specificity for DWI and low sensitivity for ASL. This is likely due to the wide time range of performing the MRI. In addition, due to instrumental limitations, the slice thickness of the MRI scan used was thicker. Finally, only a few available followup data were acquired from these babies, and further analysis is needed to be performed.

\section{Conclusions}

Our findings show that ASL noninvasively reflects the CBF changes, while DWI demonstrates the early diffusion changes in brain tissues. These two techniques could provide more detailed information on the pathological processes of HIE.

\section{Abbreviations}

ASL, arterial spin-labeled; DWI, diffusion-weighted imaging; T1WI, T1-weighted imaging; T2WI, T2-weighted imaging; HIE, hypoxic-ischemic encephalopathy; CBF, cerebral blood flow; ADC, apparent diffusion coefficient; MRI, Magnetic resonance imaging; PASL, pulse arterial spin labeling; CASL, continuous arterial spin labeling; SNR, signal-tonoise ratio.

\section{Author contributions}

LM, QW, YL, XXM and WL collected and analyzed the data. LM interpreted the data and drafted the article. QW designed the study and revised it critically for important intellectual content. All authors have read and approved the manuscript.

\section{Ethics approval and consent to participate}

The Ethics Committee of Qilu Hospital of Shandong University approved this work (ethical approval number: 12184). In all cases, informed consent was obtained from the parents of all neonates.

\section{Acknowledgment}

Not applicable. 


\section{Funding}

This research received no external funding.

\section{Conflict of in terest}

The authors declare no conflict of interest.

\section{References}

[1] Douglas-Escobar M, Weiss MD. Hypoxic-Ischemic Encephalopathy. JAMA Pediatrics. 2015; 169: 397.

[2] Massaro AN, Bouyssi-Kobar M, Chang T, Vezina LG, du Plessis AJ, Limperopoulos C. Brain perfusion in encephalopathic newborns after therapeutic hypothermia. American Journal of Neuroradiology. 2013; 34: 1649-1655.

[3] Wintermark P, Hansen A, Gregas MC, Soul J, Labrecque M, Robertson RL, et al. Brain perfusion in asphyxiated newborns treated with therapeutic hypothermia. American Journal of Neuroradiology. 2011; 32: 2023-2029.

[4] Barkovich AJ, Westmark K, Partridge C, Sola A, Ferriero DM. Perinatal asphyxia: MR findings in the first 10 days. American Journal of Neuroradiology. 1995; 16: 427-438.

[5] Badve CA, Khanna PC, Ishak GE. Neonatal ischemic brain injury: what every radiologist needs to know. Pediatric Radiology. 2012 42: 606-619.

[6] Rutherford M, Ward P, Allsop J, Malamateniou C, Counsell S. Magnetic resonance imaging in neonatal encephalopathy. Early Human Development. 2005; 81: 13-25.

[7] Tanner SF. Cerebral perfusion in infants and neonates: preliminary results obtained using dynamic susceptibility contrast enhanced magnetic resonance imaging. Archives of Disease in Childhood - Fetal and Neonatal Edition. 2003; 88: 525F-5530.

[8] Tortora D, Mattei PA, Navarra R, Panara V, Salomone R, Rossi A, et al. Prematurity and brain perfusion: Arterial spin labeling MRI. NeuroImage Clinical. 2017; 15: 401-407.

[9] De Vis JB, Hendrikse J, Petersen ET, de Vries LS, van Bel F, Alderliesten T, et al. Arterial spin-labelling perfusion MRI and outcome in neonates with hypoxic-ischemic encephalopathy. European Radiology. 2015; 25: 113-121.

[10] Alderliesten T, de Vries LS, Benders MJ, Koopman C, Groenendaal F. MR imaging and outcome of term neonates with perinatal asphyxia: value of diffusion-weighted MR imaging and (1)H MR spectroscopy. Radiology. 2011; 261: 235-242.

[11] Barkovich AJ, Miller SP, Bartha A, Newton N, Hamrick SEG, Mukherjee $\mathrm{P}$, et al. MR imaging, MR spectroscopy, and diffusion tensor imaging of sequential studies in neonates with encephalopathy. American Journal of Neuroradiology. 2006; 27: 533-547.

[12] Rutherford M, Counsell S, Allsop J, Boardman J, Kapellou O, Larkman D, et al. Diffusion-weighted magnetic resonance imaging in term perinatal brain injury: a comparison with site of lesion and time from birth. Pediatrics. 2004; 114: 1004-1014.

[13] Twomey E, Twomey A, Ryan S, Murphy J, Donoghue VB. MR imaging of term infants with hypoxic-ischemic encephalopathy as a predictor of neurodevelopmental outcome and late MRI appearances. Pediatric Radiology. 2010; 40: 1526-1535.

[14] Executive summary: Neonatal encephalopathy and neurologic outcome, second edition. Report of the American College of Obstetricians and Gynecologists Task Force on Neonatal Encephalopathy. Obstetrics and Gynecology. 2014; 123: 896-901.

[15] Detre JA, Leigh JS, Williams DS, Koretsky AP. Perfusion imaging. Magnetic Resonance in Medicine. 1992; 23: 37-45.

[16] Roberts DA, Detre JA, Bolinger L, Insko EK, Leigh JS. Quantitative magnetic resonance imaging of human brain perfusion at $1.5 \mathrm{~T}$ using steady-state inversion of arterial water. Proceedings of the National Academy of Sciences of the United States of America. 1994; 91: 33-37.
[17] Liu TT, Brown GG. Measurement of cerebral perfusion with arterial spin labeling: Part 1. Methods. Journal of the International Neuropsychological Society. 2007; 13: 517-525.

[18] Miranda MJ, Olofsson K, Sidaros K. Noninvasive measurements of regional cerebral perfusion in preterm and term neonates by magnetic resonance arterial spin labeling. Pediatric Research. 2006; 60: 359-363.

[19] Wang J, Licht DJ. Pediatric perfusion MR imaging using arterial spin labeling. Neuroimaging Clinics of North America. 2006; 16: 149-67, ix.

[20] Wu W, Wong EC. Feasibility of velocity selective arterial spin labeling in functional MRI. Journal of Cerebral Blood Flow and Metabolism. 2007; 27: 831-838.

[21] Wang Z, Wang J, Connick TJ, Wetmore GS, Detre JA. Continuous ASL (CASL) perfusion MRI with an array coil and paralle imaging at 3T. Magnetic Resonance in Medicine. 2005; 54: 732 737.

[22] Dai W, Garcia D, de Bazelaire C, Alsop DC. Continuous flowdriven inversion for arterial spin labeling using pulsed radio frequency and gradient fields. Magnetic Resonance in Medicine. 2008; 60: 1488-1497.

[23] L'Abee C, de Vries LS, van der Grond J, Groenendaal F. Early diffusion-weighted MRI and 1H-Magnetic Resonance Spectroscopy in asphyxiated full-term neonates. Biology of the Neonate. 2005; 88: 306-312.

[24] Perlman JM. Summary proceedings from the neurology group on hypoxic-ischemic encephalopathy. Pediatrics. 2006; 117: S28S33.

[25] Chau V, Poskitt KJ, Miller SP. Advanced Neuroimaging Techniques for the Term Newborn with Encephalopathy. Pediatric Neurology. 2009; 40: 181-188.

[26] Robertson RL, Ben-Sira L, Barnes PD, Mulkern RV, Robson CD Maier SE, et al. MR line-scan diffusion-weighted imaging of term neonates with perinatal brain ischemia. American Journal of Neuroradiology. 1999; 20: 1658-1670.

[27] Wintermark P, Moessinger AC, Gudinchet F, Meuli R. Temporal evolution of MR perfusion in neonatal hypoxic-ischemic encephalopathy. Journal of Magnetic Resonance Imaging. 2008; 27: 1229-1234.

[28] Thornton C, Rousset CI, Kichev A, Miyakuni Y, Vontell R, Baburamani AA, et al. Molecular mechanisms of neonatal brain injury. Neurology Research International. 2012; 2012: 506320.

[29] Winter JD, Lee DS, Hung RM, Levin SD, Rogers JM, Thompson $\mathrm{RT}$, et al. Apparent diffusion coefficient pseudonormalization time in neonatal hypoxic-ischemic encephalopathy. Pediatric Neurology. 2007; 37: 255-262.

[30] Marchal G, Young AR, Baron JC. Early postischemic hyperperfusion: pathophysiologic insights from positron emission tomography. Journal of Cerebral Blood Flow and Metabolism. 1999; 19: 467-482.

[31] Pienaar R, Paldino MJ, Madan N, Krishnamoorthy KS, Alsop DC, Dehaes M, et al. A quantitative method for correlating observations of decreased apparent diffusion coefficient with elevated cerebral blood perfusion in newborns presenting cerebral ischemic insults. NeuroImage. 2012; 63: 1510-1518.

[32] Aslan S, Lu H. On the sensitivity of ASL MRI in detecting regional differences in cerebral blood flow. Magnetic Resonance Imaging. 2010; 28: 928-935.

[33] Liauw L, van Wezel-Meijler G, Veen S, van Buchem MA, van der Grond J. Do apparent diffusion coefficient measurements predict outcome in children with neonatal hypoxic-ischemic encephalopathy? American Journal of Neuroradiology. 2009; 30: 264-270.

[34] Rutherford M, Malamateniou C, McGuinness A, Allsop J, Biarge MM, Counsell S. Magnetic resonance imaging in hypoxicischaemic encephalopathy. Early Human Development. 2010; 86: 351-360. 